\title{
Road Network Analysis for Ambulance Transportation Service Using Geographical Information System (GIS): A Case of Arada Sub-City, Addis Ababa, Ethiopia
}

\author{
Mikir Kassaw ${ }^{*}$, Bedasa Asefa \\ Department of Surveying Engineering, Debre Berhan University, Debre Berhan, Ethiopia \\ Email address: \\ mikir.kassaw8@gmail.com (M. Kassaw) \\ ${ }^{*}$ Corresponding author \\ To cite this article: \\ Mikir Kassaw, Bedasa Asefa. Road Network Analysis for Ambulance Transportation Service Using Geographical Information System (GIS): \\ A Case of Arada Sub-City, Addis Ababa, Ethiopia. American Journal of Traffic and Transportation Engineering. \\ Vol. 5, No. 6, 2020, pp. 65-77. doi: 10.11648/j.ajtte.20200506.11
}

Received: November 27, 2020; Accepted: December 10, 2020; Published: December 31, 2020

\begin{abstract}
Optimum utilization of time after an accident which is golden hour serves as a measure of effectiveness of ambulance service management system to reduce the loss of life and property. This paper presents a case study on Arada sub-city which is the oldest of all sub-cities located in the central part of the capital Addis Ababa with $11.56 \mathrm{Km}^{2}$ area wide. Its population, which is around 212,009, is highly dense and its spread of population density is about 18340 people per $\mathrm{Km}^{2}$. In the study area, the existing road network condition is not developing in line with the population growth. Accordingly, at time of disaster, the responsiveness rate of efficient ambulance transportation services to the right target area is low and unsatisfactory to level of customer's satisfaction. Therefore, the purpose of this study is to conduct road network analysis in Geographic Information System (GIS) environment for the betterment of ambulance transportation service in the sub city. A detail GIS database of the road network, ambulance location, accident locations and hospitals was prepared using Arc GIS software with the extension of network analyst. In this study, one ambulance station, three accident locations and six hospitals were used to compute $1,2 \& 4$ minutes service area and best route analyses. The impedance value $1,2 \& 4$ minute corresponds to very urgent, moderate urgent and low urgent service areas respectively. In very urgent service area domain about $33 \%$ of health centres are found whereas in moderate and low urgent service area domains about $65 \%$ and $100 \%$ of health centers are found respectively. This implies that, the current option increases health center service area domains by $34 \%$ from the existing approach. Besides, the selected sample best routes of ambulance transportation service are computed in terms of travelling time in minutes and its fuel consumption in litters. Accordingly, the estimated travelling time of the selected sample best route is about 870/30482.20 and 1138.5/3772.16 minutes/ birrs annually. This implies that, the proposed study decreases a total travelling duration and cost by 268.5/723.96 minutes/ birr from the existing one. As a result, GIS-based ambulance transportation service proto type model was developed.
\end{abstract}

Keywords: Ambulance, GIS, Impedance, Network Analyst

\section{Introduction}

Ambulance transportation service seems quite simple but in actual circumstance it is quite difficult during peak hours to save valuable human life when an accident occurs on the road network [1]. Today, road traffic accident is the cause of significant loss of human and economic resources worldwide. According to World Health Organization (WHO) nearly 3,500 people die on the world's roads every day and tens of millions of people are injured or disabled every year due to inefficient ambulance transportation service [2]. More than $85 \%$ of these casualties occur in low and middle in-come countries. It also imposes a huge economic burden on developing economies of Gross Domestic Product (GDP) in most countries including Ethiopia. One of very important factors in road accidents is medical time response. The benefit of rapid response and treatment of road accident victims are increasingly being recognized in research in road safety strategies. Recent studies indicate that improved medical response and associated technology are an important 
contributory factor to decrease in the severity of long-term injuries. It is recognized that time is a crucial factor in dealing with medical emergencies resulting from road traffic accidents. If proper first aid is given, road accident victims have a greater chance of survival and a reduction in the severity of their injuries [3]. The system of emergency medical services (EMS) in road networks is very important to decreasing response time. Each year more than 8949 road accidents occur in Addis Ababa city and road traffic crashes kill nearly 360 people, and injure or disabled 1490 more. Studies show that nearly 60 to 70 percent of all road accident deaths occur within the first few minutes at the scene or during transfer to hospital after road accidents. This is due to, high rate of population growth and with rapid increase in the number of vehicles, traffic jams, lack of footpaths and unsafe roads for people to walk or to cross [4].

In Arada Sub-city, annually, more than 868 accidents occur and nearly 31 deaths result. According to [5] traffic accident statistics data show that, around 3,841 traffic accidents 158 persons or $4.1 \%$ was death recorded in Arada sub-city. Moreover, the severity degree of disaster also paralleled increases with population growth, too. So, at time of disaster, the responsiveness rate of efficient ambulance transportation services to the right target areas will be low and unsatisfactory to level of customer's satisfaction [6]. Therefore, in the subcity the problem of ambulance transportation service is a crucial and serious time factor emergency situation. In order to eradicate this type of problems, one is always tempted to use the shortest distance route, but, this need not always the best route in ambulance emergency situations; rather shortest travel time is to be preferred over shortest distance. A shorter route does not always translate to shorter travel time, because it may be narrow in width or it may have higher volume of traffic or more numbers of signals and turns, type of slope road, road width, and number of junctions and so on will be analysed and determining the optimal route having the minimum travel time.

Geographic Information Systems (GISs) have been presented as a powerful analysing tool of solving complicated road networks [7]. Integration of GIS and transportation has led to a trend of analysis, decision making and the implementations of projects which can be done faster and with more confidence [8]. Application of GIS in better and more optimized designation of best route finding, determination of black spots and nearest emergency hospitals in road accidents which is an aspect that most people use today [9]. In this study, GIS-based prototype for ambulance transportation service was conducted using Network Analyst Extension in Arc GIS environment. This proposed prototype will try to solve the routing problem of ambulance transportation service (ambulance station - accident scenenearest emergency hospital) in the sub-city [10]. GIS gives us the power to create maps, integrate information, visualize scenarios, solve complicated problems, present powerful ideas, and develop effective solutions [11]. Most commercial GIS software vendors have extended their basic GIS data model during the past two decades to incorporate these innovations [12]. Finally, ambulance transportation service proto type model was developed using GIS software and has been evaluated by GIS users [13].

Therefore, the main aim of this study is to conduct road network analysis in GIS environment for the improvement of ambulance transportation service according to black spot and emergency hospital information in Arada sub-city as a case and model study. Specifically locating all the demand and supply nodes of ambulance transportation service; conducting service area analysis for generating service area boundaries of each supply nodes in the sub city; perform best route analysis for selecting routes with minimum impedance from the supply node to the selected demand nodes in the sub city and comparing the service boundaries of health centers and the traveling time of the selected best routes of the two techniques.

\section{Methodology of the Study}

\subsection{Materials Used}

In this study, different materials such as hardware's and software's were used to achieve the objectives of the study as indicated table 1 .

Table 1. List of materials.

\begin{tabular}{|c|c|c|c|}
\hline No & Hardware/software & Company & Purpose/for \\
\hline \multirow{4}{*}{1} & Handheld GPS & Garmin & collecting Ground Control Points (GCPs) \\
\hline & Laptop & Toshiba & conducting the general activities \\
\hline & Scanner (A1) & HP & converting hard copy to soft copy \\
\hline & Printer & HP Laser-Jet & printing documents \\
\hline 2 & $\begin{array}{l}\text { ArcGIS (version 9.3) } \\
\text { MS-office, (like MS-Excel (version 2010) }\end{array}$ & $\begin{array}{l}\text { ESRI } \\
\text { Microsoft }\end{array}$ & $\begin{array}{l}\text { building geo-database and network analysis } \\
\text { turn feature calculation }\end{array}$ \\
\hline
\end{tabular}

\subsection{Methods}

The existing road network of Arada sub city across Addis Ababa has got problems related to efficient ambulance transportation service at time of disaster [14]. This section will focus on the methods and data's required to analyze and evaluate the current and conventional approaches of ambulance transportation service using GIS software to come up with the right solutions for the improvement of ambulance transportation service based on specific travel impedance.

Before collecting the data, the type of data that can be used for further analysis could be classified. So, in this study, two types of data were used (i.e., primary and secondary data) to carry out the objectives.

\section{Primary data}

The following data collection methods were used. About 
$10 \%$ of the primary research data was collected through interview. The rest $90 \%$ was collected through field survey. Interview was employed to collect data related to ambulance transportation system, places which are frequently happened accidents were conducted in different government officials and professionals. Field survey was the dominant method of the primary data collection. Ambulance station, black spots and nearby emergency hospital locations were collected in the field using Handheld GPS. Moreover, the geographic coordinates of the study area was also gathered same manner in the field for the purpose of geo-referencing.

\section{Secondary data}

Road network data was collected in the form of hard copy from Ethiopian Mapping Agency (EMA) to develop the database for the road networks. Mainly four types of attribute data were collected from the road network map. First are descriptor attributes, which gives the description like name of road, road class, speed limit and elevation value. Second are the cost attributes, which play the essential role in the analysis of road network to find the best route which has the cost of length of road in meters and cost of drive time in minutes. Third are the restriction attributes, which also have the main role accompanying with cost attributes. This attribute data include restriction value like one-way, U-turns, traffic light and others. In this study, there is only one restriction attribute that is one-way road that was considered to set its value on the respective roads. And fourth are hierarchy attributes which identifies the type of roads and give their hierarchy from primary to local roads to perform network analysis by using a hierarchy.

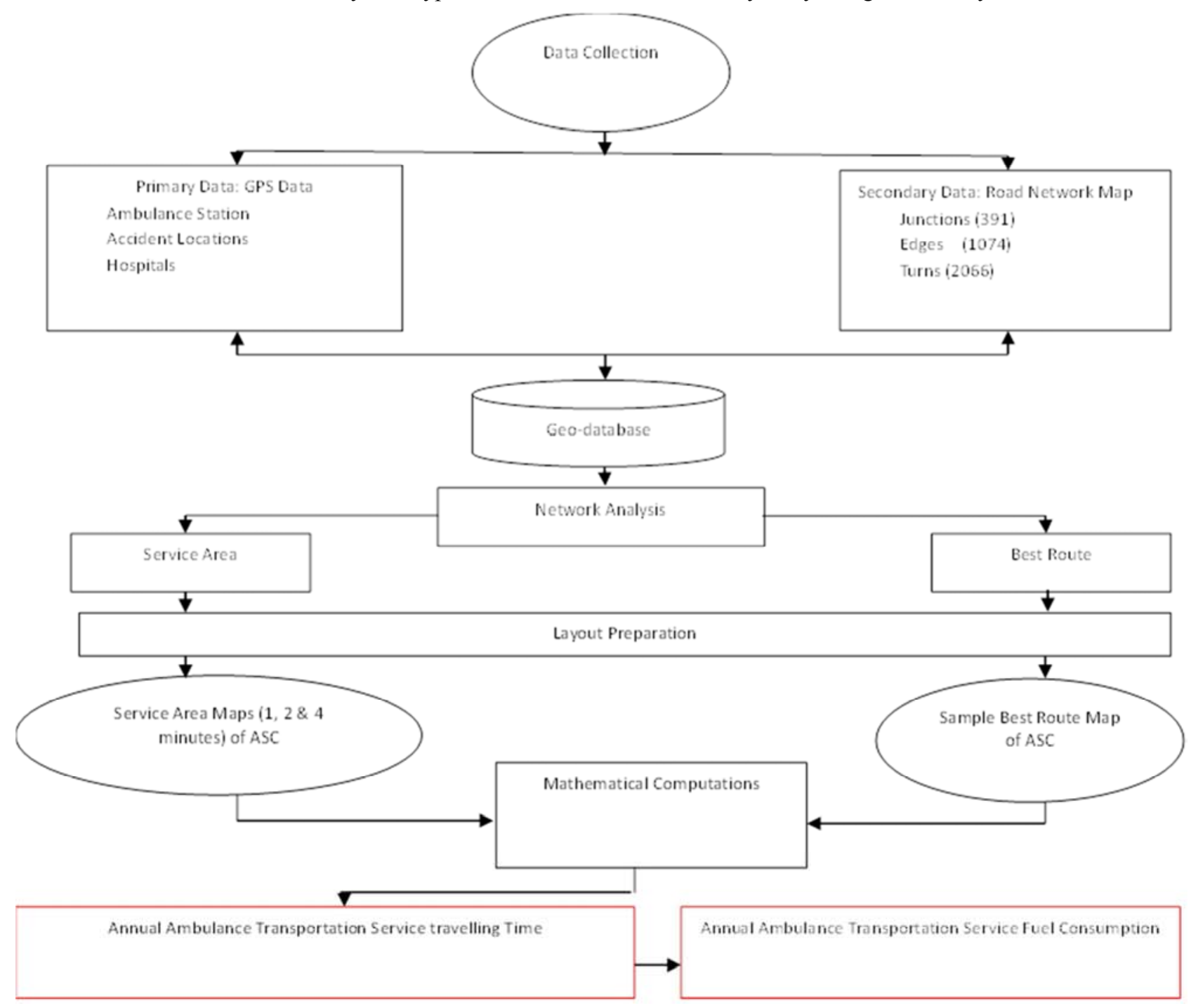

Figure 1. Technological schemes of ambulance transportation service.

\section{Data Analysis, Results and Discussions}

\subsection{Data Analysis and Results}

The study on network analysis for ambulance emergency service at Arada sub-city was carried out in GIS environment by applying network analyst extension. In this study, the identified accidental sites (i.e., Semen Hotel Mebrat, Parlama Mebrat and Kebena Mebrat) in Arada sub-city were the target sites for ambulance services. Moreover, the ambulance station (i.e., Arada branch) and Hospitals (i.e., Ras Desta, Tibebu General, Yekatit 12, Menillik II, Zewuditu and 
Ghandi hospitals) were also spots where ambulance route cover in due to time the emergency services in the sub-city. In addition, the type of accidents were assumed to be the highly emergent (one minute), moderately emergent (two minutes) and low emergent (four minutes) for the different scenarios of the three identified black spots in the study area. Therefore, the analyses on ambulance service in the sub city were categorized into two as service area analysis and best route analysis.

\subsubsection{Service Area Analysis}

This analysis in network analysis environment computes service area polygon that encompasses all the area within the reach of the located hospitals. Accordingly the above the mentioned one, two and four minutes service area domains were generated for all the identified accident locations in the sub city. This could be done by adding network dataset to the
Arc Map and selecting service area analysis from the drop down menu, and then load that the hospital points into the facilities list on the network analyst window and solve the three minutes service area analysis [15] as described in the following sub sections.

\section{One minute service area analysis scenarios}

Except Semen Hotel Mebrat accident site, all the rest sites (i.e., parlama mebrat and kebena mebrat) are not accessible to the existing health centers in one minute service area domains in the sub-city. The result showed that, Semen Hotel Mebrat accident site was accessible to two health centers from the six health centers which are found in the sub city. Accordingly, about $33 \%$ of health centers are found in one minute service area domain. This implies that, highly accidents could be addressed only for Semen Hotel Mebrat accident site as shown below in table 2 and figure 2 .

Table 2. One minute service area analysis.

\begin{tabular}{|c|c|c|c|c|}
\hline Accident Location & Service area & Accessible HC & Names & Percentage \\
\hline SHM & 1 & 2 & Ras Desta Tibebu & $(2 / 6 * 100)=33 \%$ \\
\hline PM & 1 ' & 0 & None & 0 \\
\hline KM & 1 , & 0 & None & 0 \\
\hline Total & & & & $(2 / 6 * 100)=33 \%$ \\
\hline
\end{tabular}

*HC: Health Center, *SHM: Semen Hotel Mebrat, PM: Parlama Mebrat \& KM: Kebena Mebrat.

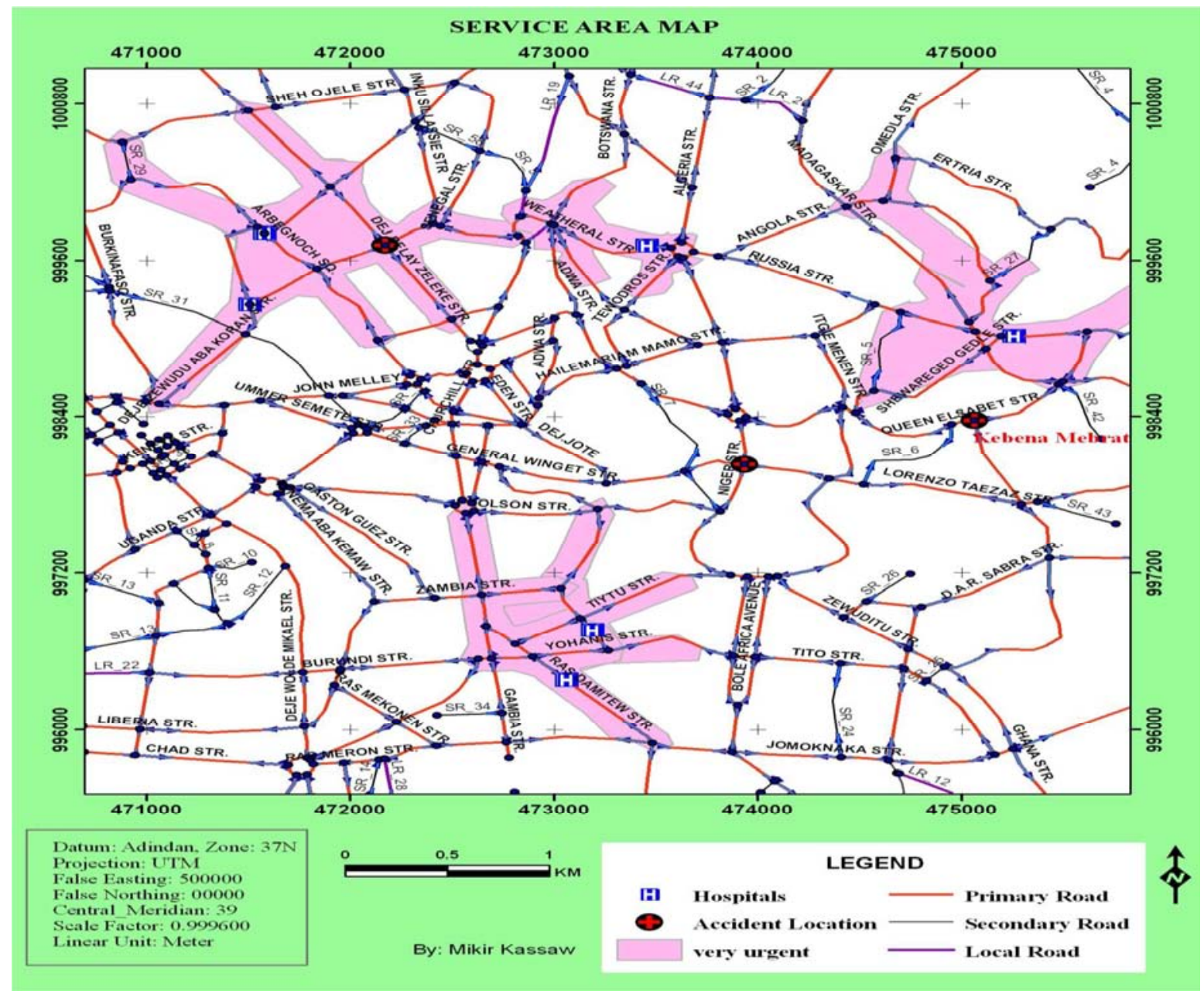

Figure 2. One minute health center service area map of Arada Sub-City. 


\section{Two minute service area analysis scenarios}

All accident sites (i.e., semen hotel mebrat, parlama mebrat and kebena mebrat) are accessible to the existing health centers in the sub-city. The result showed that, semen hotel mebrat accident site was accessible to two health centers, parlama mebrat and kebena mebrat accident sites were accessible to one health center for each. According to table 2 and figure 2 shows that, about $65 \%$ of health centers are found in two minute service area domain. This implies that, moderately accidents could be addressed for all accident sites in the sub-city.

Table 3. Two minute service area analysis.

\begin{tabular}{lllll}
\hline Accident Location & Service Area & Accessible HC & Names & Percentage \\
\hline SHM & 2 & 2 & Ras Desta, Tibebu & $\left(2 / 6^{*} 100\right)=33 \%$ \\
PM & 2, & 1 & Zewuditu & $\left(1 / 6^{*} 100\right)=16 \%$ \\
KM & 2, & 1 & Menillik II & $\left(1 / 6^{*} 100\right)=16 \%$ \\
Total & & & & $\left(4 / 6^{*} 100\right)=65 \%$ \\
\hline
\end{tabular}

*HC: Health Center, ${ }^{*}$ SHM: Semen Hotel Mebrat, PM: Parlama Mebrat \& KM: Kebena Mebrat.

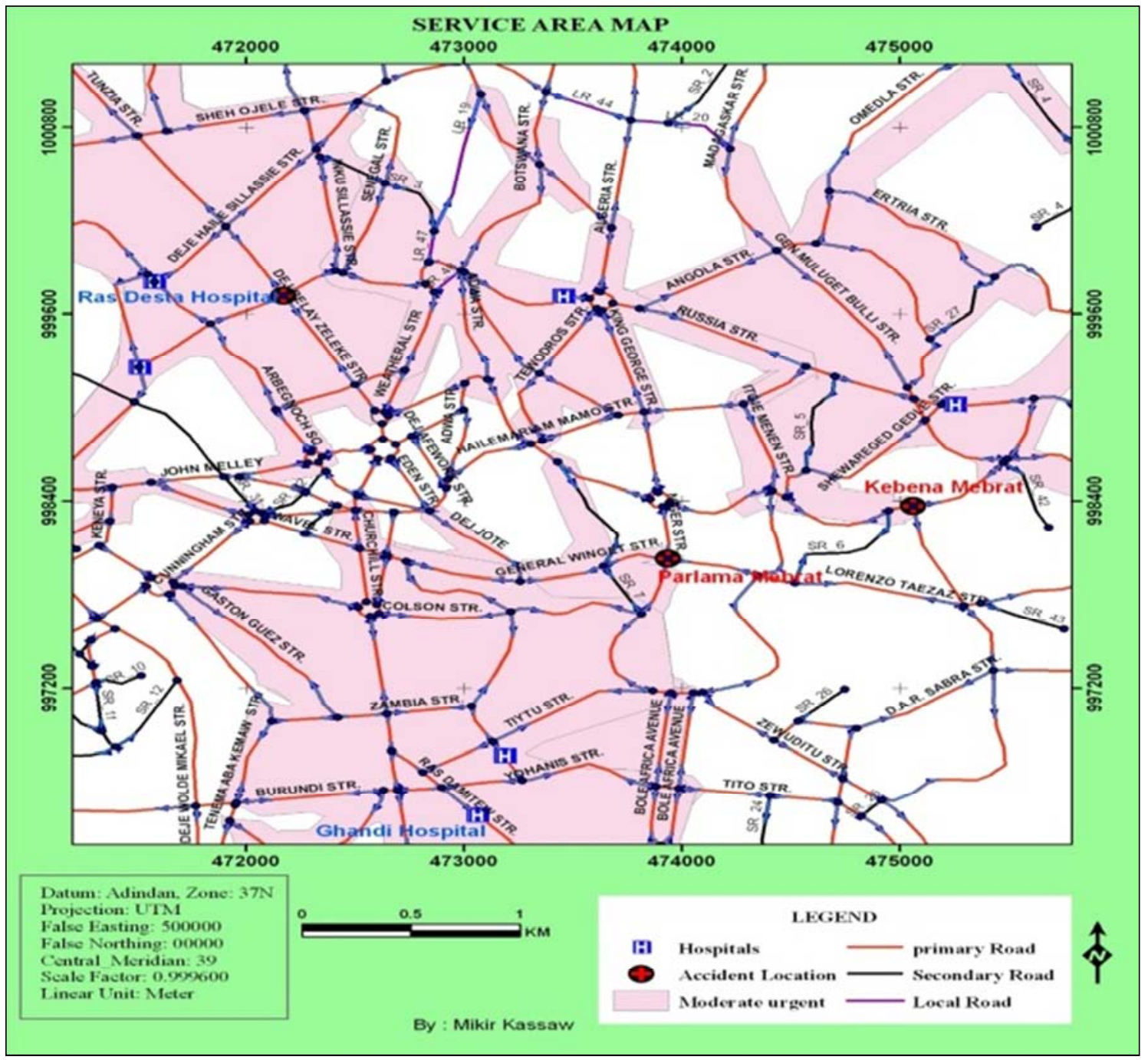

Figure 3. Two minute health center service area map of Arada Sub-City.

III. Four minute service area analysis scenarios

In this analysis, all accident sites (i.e., semen hotel mebrat, parlama mebrat and kebena mebrat) are accessible to all the existing health centers in the sub-city. The result showed that, semen hotel mebrat accident site was accessible to three health centers, parlama mebrat accident site was accessible to four health centers and kebena mebrat accident site was also accessible to four health centers. According to table 3 and figure $3,100 \%$ of health centers are found in four minute service area domain. This implies that, Low accidents could be addressed for all accident sites in the sub-city. 
Table 4. Four minute service area analysis.

\begin{tabular}{lllll}
\hline Accident Location & Service Area & Accessible HC & Names & Percentage \\
\hline SHM & 4 & 3 & Ras Desta, Tibebu, Yekatit & $(3 / 6 * 100)=50 \%$ \\
PM & 4 & 4 & Zewuditu, Yekatit 12, Menilik II, Ghandi & $(4 / 6 * 100)=66 \%$ \\
KM & 4, & 4 & Menilik II, Yekatit 12, Zewuditu, Ghandi & $(4 / 6 * 100)=66 \%$ \\
Total & & & & $(6 / 6 * 100)=100 \%$ \\
\hline
\end{tabular}

*HC: Health Center, SHM: Semen Hotel Mebrat, PM: P arlama Mebrat \& KM: Kebena Mebrat.

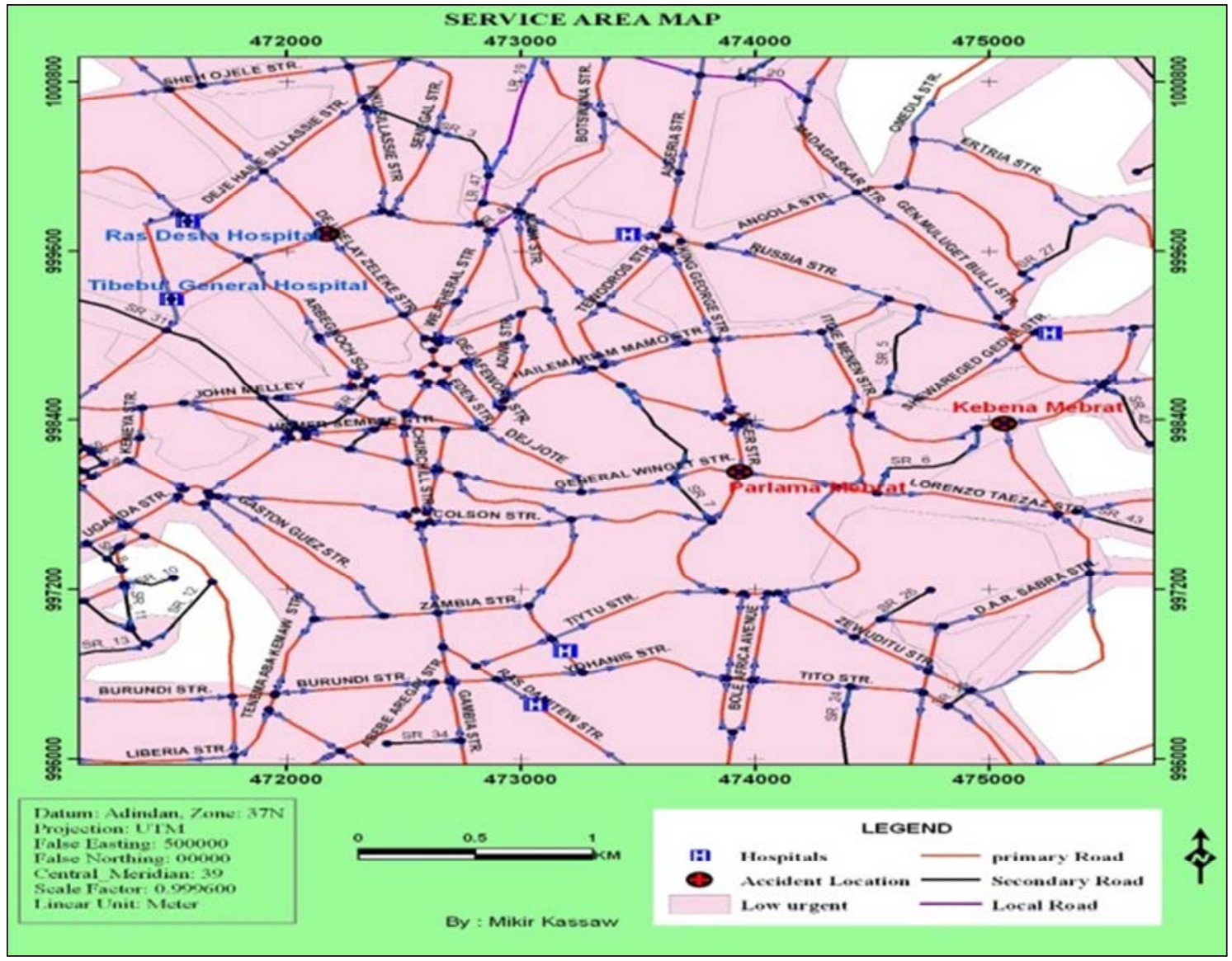

Figure 4. Four minute health center service area map of Arada Sub-City.

\section{Comparison of Service Area Analysis}

This study has come up with two approaches for service area domain comparisons in specific and in general terms. The former approach indicates (i.e., specific term) the peer to peer comparisons of individual black spots from the proposed and existing approaches point of view. The later one (i.e., general term) focuses on the cumulative service area domain comparisons of the proposed and existing approaches. For specific comparison considering Semen Hotel Mebrat as a selected accident location, the total number of accessible health centers to address Semen Hotel Mebrat accident site is three in the proposed approach and two in the existing one. This implies that, $50 \%$ and $33 \%$ of health centers were accessible in four minute service area analysis in the proposed and existing approaches respectively. Consequently, the difference in accessibility between the two (i.e., proposed and existing approaches) for Semen Hotel Mebrat accident site is $17 \%$. This indicates that, the proposed approach maximizing service area domain by increasing $17 \%$ from the existing one. Besides, it maximizes the benefits of ambulance service by increasing 33\% and 50\% service area domain for Parlama Mebrat and Kebena Mebrat accident site respectively. For cumulative comparisons considering all accident sites as a selected accident location, the total numbers of health centers to address all accident sites (i.e., Semen Hotel Mebrat, Parlama Mebrat and Kebena Mebrat) are six in the proposed approach and four in the exiting one. This illustrates that, $100 \%$ and $66 \%$ of health centers were accessible in the same minute (four) service area analysis in the proposed and existing approaches respectively. Accordingly, the difference shows that, the proposed approach maximizing service area domain by increasing $34 \%$ from the existing one. This implies that, the current study come up with a total increasing of service area domain by $34 \%$ from the existing approach. 
Table 5. Comparison of service area analysis.

\begin{tabular}{|c|c|c|c|c|c|}
\hline Methods & Accident Location & Service area & Accessible HC & Names & percentage \\
\hline \multirow{6}{*}{ Proposed } & & $1 ’ \& 2$ & 2 & Ras Desta, Tibebu & \multirow{2}{*}{$(3 / 6) * 100=50$} \\
\hline & SHM & 4 & 3 & Ras Desta, Tibebu, yekatit 12 & \\
\hline & \multirow{2}{*}{ PM } & 2 ' & 1 & Zewuditu & \multirow{2}{*}{$(4 / 6) * 100=66$} \\
\hline & & 4, & 4 & Zewuditu, Yekatit 12, Menilik II, Ghandi & \\
\hline & \multirow{2}{*}{ KM } & $2^{\prime}$ & 1 & Menillik II & \multirow{2}{*}{$(4 / 6) * 100=66$} \\
\hline & & 4 ' & 4 & Menilik II, Yekatit 12, Zewuditu, Ghandi & \\
\hline \multirow{5}{*}{ Existing } & \multirow{2}{*}{ SHM } & 1'\&2' & 1 & Ras Desta & \multirow{2}{*}{$(2 / 6) * 100=33$} \\
\hline & & 4 & 2 & Ras Desta, Yekatit 12 & \\
\hline & \multirow{2}{*}{ PM } & 2 ' & 1 & Zewuditu & \multirow{2}{*}{$(2 / 6) * 100=33$} \\
\hline & & 4 ' & 2 & Zewuditu, yekatit 12 & \\
\hline & KM & $2^{\prime} \& 4{ }^{\prime}$ & 1 & Menilik II & $(1 / 6) * 100=16$ \\
\hline
\end{tabular}

\subsubsection{Best Route Analysis}

This analysis in network analysis environment selects best routes from the ambulance station to accident location and then to the nearest emergency hospitals again depends on the time constraints [9]. In this analysis, three different best routes (i.e., one, two and four minutes) for each accident sites (i.e., Semen Hotel Mebrat, Parlama Mebrat and Kebena Mebrat) were made in the sub-city. This could be done by adding network dataset to the Arc Map and selecting Best Route analysis from the drop down menu, and then load that the origin and destination points into the stops list on the network analyst window and solve the quickest and shortest path routes from the origin to the destination as described in the following sub sections.

\section{Best Route Analysis for SHM accident site}

The best route for Semen Hotel Mebrat accident site was computed in network analysis environment by considering Semen Hotel Mebrat accident site, hospitals and ambulance stations as stops feature layer; congested areas where ambulance can't travel as barrier feature layer and generated routes from ambulance station - accident location-the nearest emergency hospitals as route feature layer as indicated in table 6 and figure 5 below. Moreover, the computation of best route was determined time as impedance for the overall road networks of the sub-city. This implies that, the generated route that extends from ambulance station to Semen Hotel Mebrat accident site; and from there to the nearest emergency hospital (i.e., Ras Desta Hospital) is the quickest path (first best route) as shown in purple and has a total length of 3.2 $\mathrm{km}$ which takes 2-minutes to traverse.

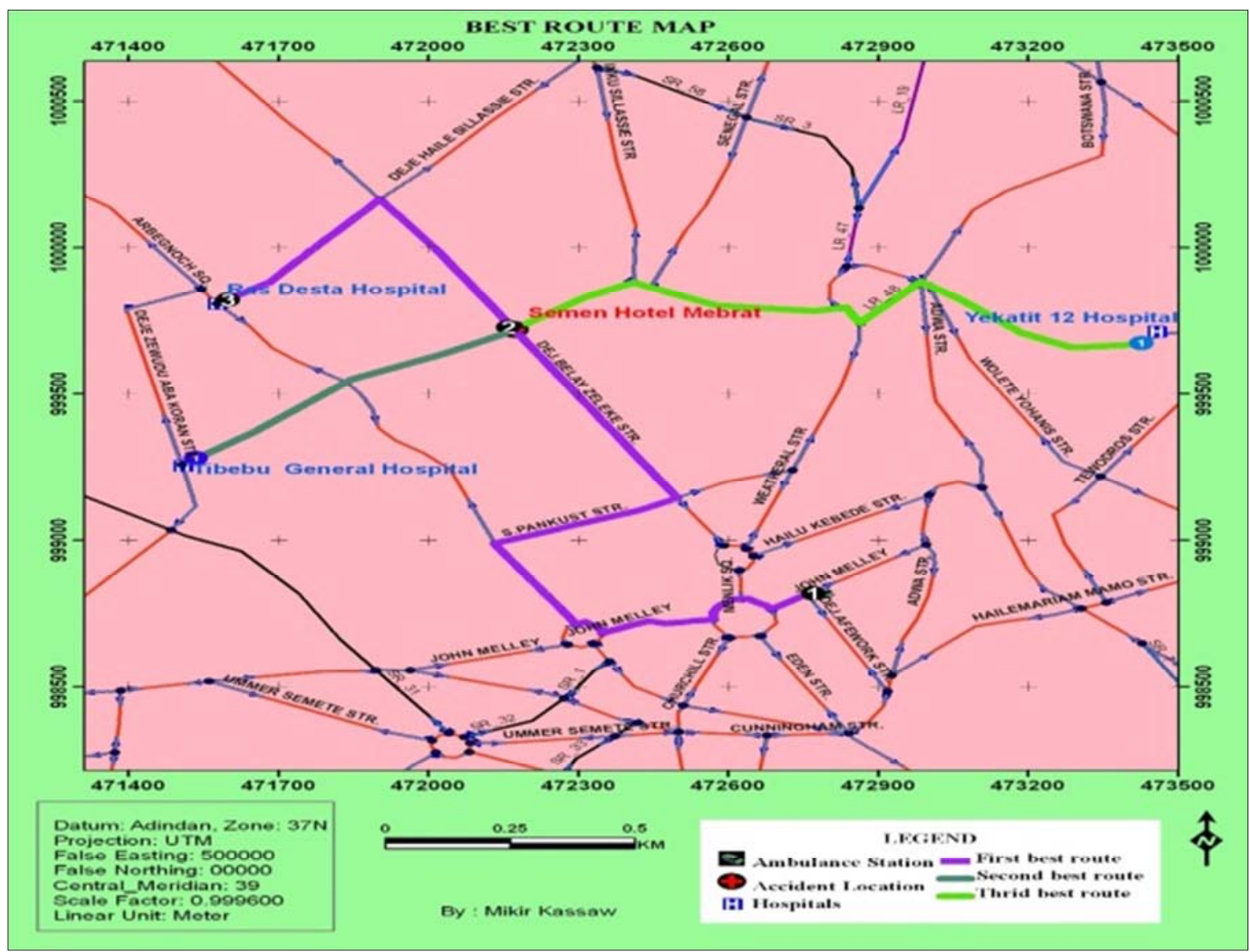

Figure 5. Best route map from ambulance station -SHM accident - hospitals. 
Table 6. Best route annual traveling time cost analysis for SHM accident site.

\begin{tabular}{|c|c|c|c|c|c|c|c|c|c|}
\hline \multirow{2}{*}{ AS } & \multirow{2}{*}{$\mathrm{HC}$} & \multicolumn{2}{|c|}{ Best Route from AS - AS - HC } & \multirow{2}{*}{$\mathbf{F}$} & \multirow{2}{*}{$\begin{array}{l}\text { DP (Oct } \\
12,2012)\end{array}$} & \multirow{2}{*}{$\begin{array}{l}\mathrm{L}=\mathrm{D} / 5.5 \mathrm{k} \\
\mathrm{m}\end{array}$} & \multirow{2}{*}{$\mathbf{S T P}=L * D P$} & \multicolumn{2}{|c|}{ Cost in terms of } \\
\hline & & D (km) & $\mathbf{T}$ (min) & & & & & Time $=F * T$ & ETB $=F * S T P$ \\
\hline SHM & Ras Desta & 3.2 & 2 & 30 & 16.91 & 0.58 & 9.84 & 60 & 295.16 \\
\hline
\end{tabular}

\section{Best Route Analysis for PM accident site}

The best route for Parlama Mebrat accident site was computed in network analysis environment by considering Parlama Mebrat accident site, hospitals and ambulance stations as stops feature layer; congested areas where ambulance can't travel as barrier feature layer and generated routes from ambulance-accident-hospital as route feature layer as indicated in table 7 and figure 6.
Moreover, the computation of best route was determined by using time as impedance for the overall road networks of the sub-city. This implies that, the generated route that extends from ambulance station to PM accident site and then to the nearest emergency hospital (i.e., Zewuditu Hospital) is the quickest path as shown in purple and has a total length of $4.2 \mathrm{~km}$ which takes 3 -minutes to traverse.

Table 7. Best route annual traveling time cost analysis for PM accident site.

\begin{tabular}{|c|c|c|c|c|c|c|c|c|c|}
\hline \multirow{2}{*}{ AS } & \multirow{2}{*}{ HC } & \multicolumn{2}{|c|}{ Best Route from AS- AS -HC } & \multirow{2}{*}{$\mathbf{F}$} & \multirow{2}{*}{$\begin{array}{l}\text { DP (Oct 12, } \\
\text { 2012) }\end{array}$} & \multirow{2}{*}{$\mathbf{L}=\mathbf{D} / 5.5 \mathrm{~km}$} & \multirow{2}{*}{$\mathbf{S T P}=\mathbf{L} * \mathbf{D P}$} & \multicolumn{2}{|c|}{ Costs in terms of } \\
\hline & & $\mathrm{D}(\mathrm{Km})$ & T (Min) & & & & & Time $=F * T$ & $\mathbf{E T B}=\mathbf{F} * \mathbf{S T P}$ \\
\hline PM & Zewuditu & 4.2 & 3 & 45 & 16.91 & 0.76 & 12.91 & 135 & 581.09 \\
\hline
\end{tabular}

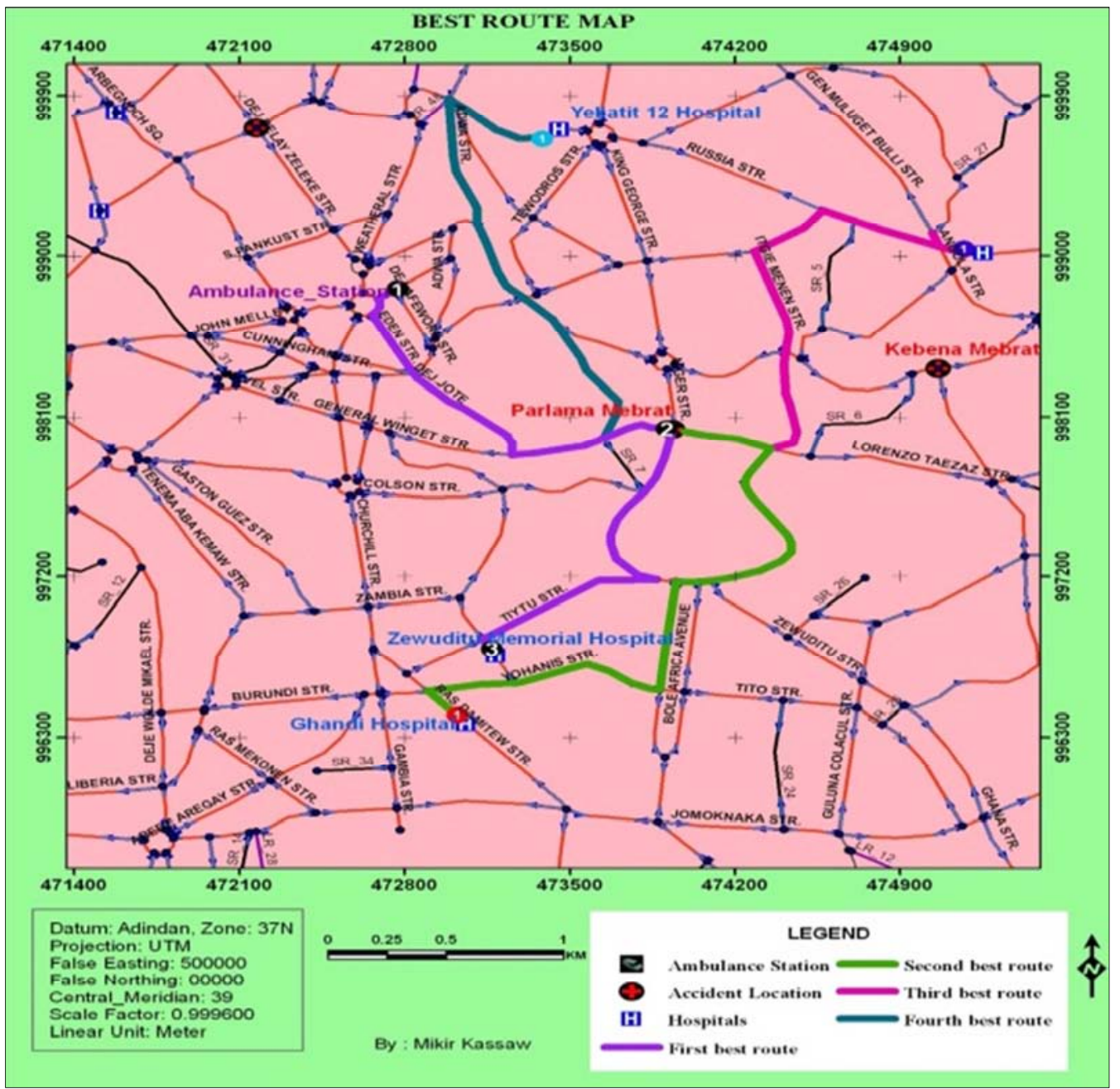

Figure 6. Best route map from ambulance station - PM accident-hospitals.

\section{Best Route Analysis for KM accident site}

The best route for accident site was computed in network analysis environment by considering Kebena Mebrat accident site, hospitals and ambulance stations as stops feature layer; congested areas where ambulance can't travel as barrier feature layer and generated routes from ambulance-accidenthospital as route feature layer as indicated in table 8 and figure 7 below. Moreover, the computation of best route was determined by using time as impedance for the overall road networks of the sub-city. This implies that, the generated 
route that extends from ambulance station to Kebena Mebrat accident site and then to the nearest emergency hospital (i.e., Menillik II hospital) is the quickest path as shown in purple

and has a total length of $5.6 \mathrm{~km}$ which takes 5 minutes to travers.

Table 8. Best route annual traveling time cost analysis for KM accident site

\begin{tabular}{|c|c|c|c|c|c|c|c|c|c|}
\hline \multirow{2}{*}{ AS } & \multirow{2}{*}{ HC } & \multicolumn{2}{|c|}{ Best Route from AS - AS - HC } & \multirow{2}{*}{$\mathbf{F}$} & \multirow{2}{*}{$\begin{array}{l}\text { DP (Oct 12, } \\
\text { 2012) }\end{array}$} & \multirow{2}{*}{$\mathbf{L}=\mathbf{D} / \mathbf{5 . 5} \mathrm{km}$} & \multirow{2}{*}{$\begin{array}{l}\text { STP }=\mathbf{L} * \\
\text { DP }\end{array}$} & \multicolumn{2}{|c|}{ Costs in terms of } \\
\hline & & $\mathrm{D}(\mathrm{Km})$ & $\mathbf{T}$ (Min) & & & & & Time $=F^{*} T$ & ETB $=\mathbf{F} *$ STP \\
\hline KM & Menillik II & 5.6 & 5 & 60 & 16.91 & 1.02 & 17.22 & 300 & 1033.05 \\
\hline
\end{tabular}

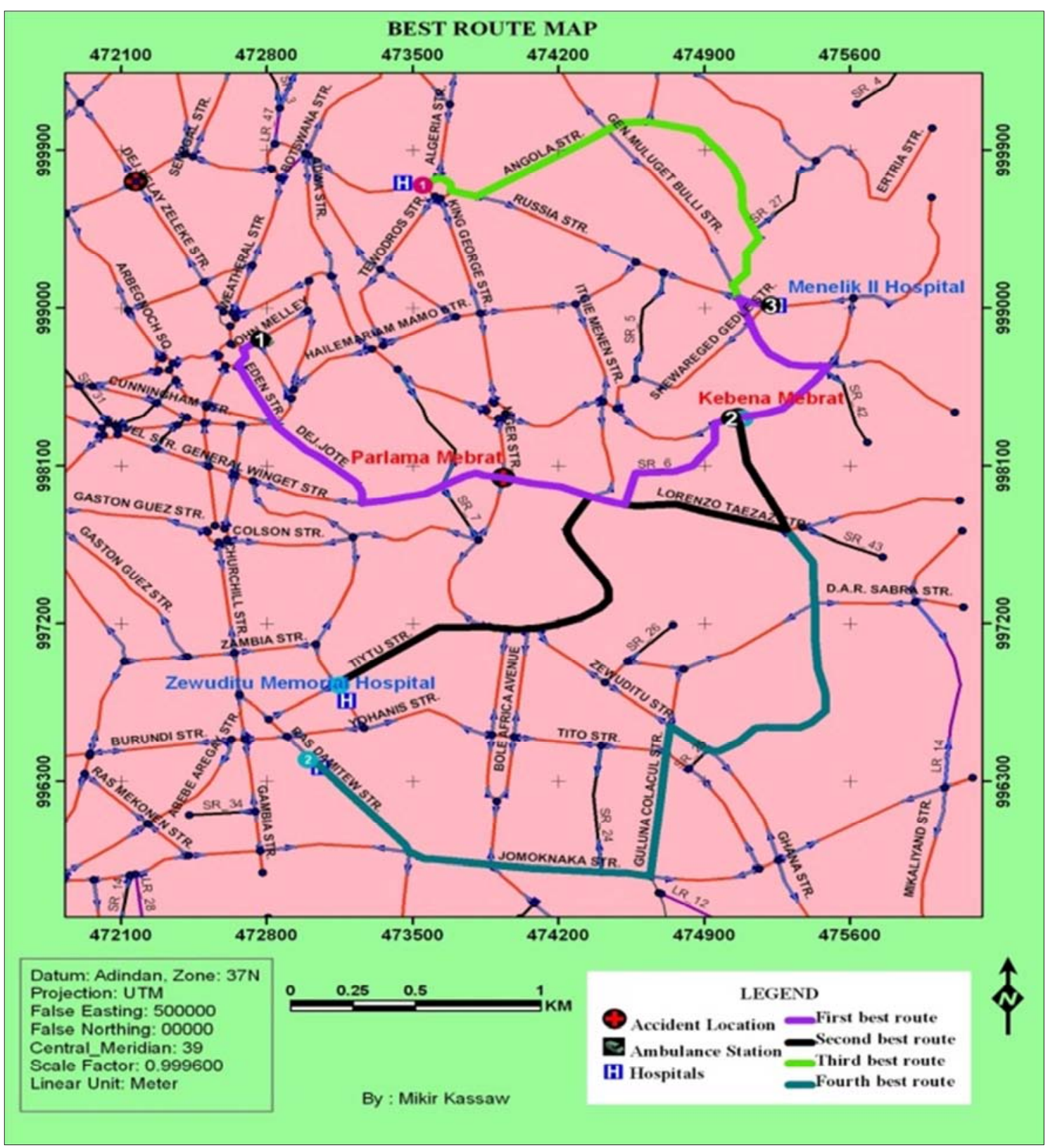

Figure 7. Best route map from ambulance station - KM accident-hospitals.

\subsubsection{Cost Evaluation in the Proposed Approach}

It is desirable to estimate the costs of the selected sample best route for utilizing real applications in the study area depend on in the proposed method.

I. Cost evaluation of best route analysis for SHM accident site

For rational decisions regarding to the feasibility of utilizing the identified best routes for practical implementation, cost evaluation is an important consideration. As per figure 4, the route from ambulance station to accident site; and from there to the nearest emergency hospital covers $3.2 \mathrm{~km}$ which takes 2 minutes to traverse for first best route; $3 \mathrm{~km}$ which takes 3 minutes for second best route and $3.8 \mathrm{~km}$ which takes 5 minutes for third best route. To sum up, the overall traversing physical 
distance is about $10 \mathrm{~km}$ and 10 minutes of travelling time. As per section 3.1.3, the estimated cost of traversing on the selected sample best route to address emergency service is about 300 minutes and 922.36 birr annually.

Table 9. Summarized annual travelling time cost analysis for SHM accident site.

\begin{tabular}{|c|c|c|c|c|c|c|c|c|c|}
\hline \multirow{2}{*}{ AS } & \multirow{2}{*}{$\mathrm{HC}$} & \multicolumn{2}{|c|}{ Best Route from AS - AS - HC } & \multirow{2}{*}{$\mathbf{F}$} & \multirow{2}{*}{$\begin{array}{l}\text { DP (Oct 12, } \\
\text { 2012) }\end{array}$} & \multirow{2}{*}{$\mathbf{L}=\mathrm{D} / 5.5 \mathrm{~km}$} & \multirow{2}{*}{$\begin{array}{l}\text { STP } \\
=\mathbf{L} * \mathbf{D P}\end{array}$} & \multicolumn{2}{|c|}{ Cost in terms of } \\
\hline & & D (km) & T (Min) & & & & & Time $=F^{*} \mathbf{T}$ & ETB $=F * S T P$ \\
\hline \multirow{3}{*}{ SHM } & Ras Desta & 3.2 & 2 & 30 & 16.91 & 0.58 & 9.84 & 60 & 295.16 \\
\hline & Tibebu & 3 & 3 & 30 & 16.91 & 0.55 & 9.22 & 90 & 276.71 \\
\hline & Yekatit 12 & 3.8 & 5 & 30 & 16.91 & 0.69 & 11.68 & 150 & 350.50 \\
\hline Total & & 10 & 10 & 90 & 50.73 & 1.82 & 30.75 & 300 & 922.36 \\
\hline
\end{tabular}

II. Cost evaluation of best route analysis for PM accident site

For coherent judgments regarding to the feasibility of utilizing the identified best routes for practical implementation, cost evaluation is an important consideration. As per figure 5, the route from ambulance station to accident site and from there to the nearest emergency hospital covers $4.2 \mathrm{~km}$ and takes 3 minutes to traverse for first best route; $4.8 \mathrm{~km}$ which takes 4 minutes for second best route; $5 \mathrm{~km}$ which takes 5 minutes for third best route and $5.7 \mathrm{~km}$ which takes 5 minutes for final best route. To sum up, the overall traversing physical distance is about $19.7 \mathrm{~km}$ and 17 minutes of travelling time. As per section 3.1.3, the estimated cost of traversing on the selected route to address emergency service is about 765 minutes and 2725.36 birr annually.

Table 10. Summarized annual traveling time cost analysis for PM accident site.

\begin{tabular}{|c|c|c|c|c|c|c|c|c|c|}
\hline \multirow{2}{*}{ AS } & \multirow{2}{*}{$\mathrm{HC}$} & \multicolumn{2}{|c|}{ Best Route from AS - AS - HC } & \multirow{2}{*}{$\mathbf{F}$} & \multirow{2}{*}{$\begin{array}{l}\text { DP (Oct 12, } \\
\text { 2012) }\end{array}$} & \multirow{2}{*}{$\mathbf{L}=\mathbf{D} / 5.5 \mathrm{~km}$} & \multirow{2}{*}{ STP $=L * D P$} & \multicolumn{2}{|c|}{ Costs in terms of } \\
\hline & & $\mathrm{D}(\mathrm{Km})$ & T (Min) & & & & & Time $=F^{*} \mathbf{T}$ & ETB $=F^{*}$ STP \\
\hline \multirow{4}{*}{ PM } & Zewuditu & 4.2 & 3 & 45 & 16.91 & 0.76 & 12.91 & 135 & 581.09 \\
\hline & Ghandi & 4.8 & 4 & 45 & 16.91 & 0.87 & 14.76 & 180 & 664.10 \\
\hline & Menelik II & 5 & 5 & 45 & 16.91 & 0.91 & 15.37 & 225 & 691.77 \\
\hline & Yekatit 12 & 5.7 & 5 & 45 & 16.91 & 1.04 & 17.52 & 225 & 788.40 \\
\hline Total & & 19.7 & 17 & 180 & 67.64 & 3.58 & 60.57 & 765 & 2725.36 \\
\hline
\end{tabular}

\section{Cost evaluation of best route analysis for KM accident} site

For real-world decisions regarding to the feasibility of utilizing the identified best routes for concrete application, cost evaluation is an important consideration. As per figure 7, the route from Ambulance station to Accident site and to the nearest emergency hospital covers $5.6 \mathrm{~km}$ which takes 5 minutes for first best route; $7.5 \mathrm{~km}$ which takes 6 minutes for second best route; $8.2 \mathrm{~km}$ which takes 7 minutes for third best route and $9.1 \mathrm{~km}$ which takes 7 minutes for final best route to traverse. To sum up, the overall traversing physical distance is about $30.4 \mathrm{~km}$ and 25 minutes of travelling time. As per section 3.1.3, the estimated cost of traversing on the selected route to address emergency service is about 1500 minutes and 5607.97 birr annually.

Table 11. Summarized annual traveling time cost analysis for KM accident site.

\begin{tabular}{|c|c|c|c|c|c|c|c|c|c|}
\hline \multirow{2}{*}{ AS } & \multirow{2}{*}{ HC } & \multicolumn{2}{|c|}{ Best Route from AS - AS - HC } & \multirow{2}{*}{$\mathbf{F}$} & \multirow{2}{*}{$\begin{array}{l}\text { DP (Oct 12, } \\
\text { 2012) }\end{array}$} & \multirow{2}{*}{$\begin{array}{l}\mathrm{L}=\mathrm{D} / 5.5 \\
\mathrm{~km}\end{array}$} & \multirow{2}{*}{$\mathbf{S T P}=\mathbf{L} * \mathbf{D P}$} & \multicolumn{2}{|c|}{ Costs in terms of } \\
\hline & & $\mathrm{D}(\mathrm{Km})$ & T (Min) & & & & & Time $=F^{*} \mathbf{T}$ & ETB $=F * S T P$ \\
\hline \multirow{4}{*}{ KM } & Menillik II & 5.6 & 5 & 60 & 16.91 & 1.02 & 17.22 & 300 & 1033.05 \\
\hline & Zewuditu & 7.5 & 6 & 60 & 16.91 & 1.36 & 23.06 & 360 & 1383.55 \\
\hline & Yekatit 12 & 8.2 & 7 & 60 & 16.91 & 1.49 & 25.21 & 420 & 1512.68 \\
\hline & Ghandi & 9.1 & 7 & 60 & 16.91 & 1.65 & 27.98 & 420 & 1678.70 \\
\hline Total & & 30.4 & 25 & 240 & 67.64 & 5.53 & 93.47 & 1500 & 5607.97 \\
\hline
\end{tabular}

\subsubsection{Cost Evaluation in the Existing Approach}

It is desirable to estimate the costs of the selected sample best route for utilizing real applications in the study area depend on conventional approaches of cost evaluation.

I. Cost evaluation of best route analysis for SHM accident site

In the existing approach, the identified best routes for utilizing practical implementation from ambulance station to
Semen Hotel Mebrat accident site; and from there to the nearest emergency hospital covers $4.3 \mathrm{~km}$ which takes 3.9 minutes to traverse for both $1 \& 2$ minutes service area and 5 $\mathrm{km}$ which takes 6.5 minutes in a four minutes service area. To sum up, the overall traversing physical distance is about 13.6 $\mathrm{km}$ and 14.3 minutes of travelling time. As per section 3.1.4, the estimated cost of traversing on the selected route to address emergency service is about 312 minutes and 857.85 birr.

Table 12. Existing annual traveling time cost analysis for SHM accident site.

\begin{tabular}{|c|c|c|c|c|c|c|c|c|c|}
\hline \multirow{2}{*}{ AS } & \multirow{2}{*}{$\mathrm{HC}$} & \multicolumn{2}{|c|}{ Best Route from AS - AS - HC } & \multirow{2}{*}{$\mathbf{F}$} & \multirow{2}{*}{$\begin{array}{l}\text { DP (Oct 12, } \\
\text { 2012) }\end{array}$} & \multirow{2}{*}{$\mathbf{L}=\mathbf{D} / \mathbf{5 . 5} \mathbf{k m}$} & \multirow{2}{*}{ STP $=L * D P$} & \multicolumn{2}{|c|}{ Cost in terms of } \\
\hline & & D (km) & $\mathbf{T}(\min )$ & & & & & Time $=F^{*} \mathbf{T}$ & ETB $=\mathbf{F} * \mathrm{STP}$ \\
\hline \multirow{2}{*}{ SHM } & Ras Desta & 4.3 & 3.9 & 30 & 16.91 & 0.782 & 13.224 & 117 & 396.72 \\
\hline & Yekatit 12 & 5 & 6.5 & 30 & 16.91 & 0.909 & 15.371 & 195 & 461.13 \\
\hline Total & & 9.3 & 10.4 & 60 & 33.38 & 1.691 & 28.595 & 312 & 857.85 \\
\hline
\end{tabular}




\begin{abstract}
II. Cost evaluation of best route analysis for PM accident site

In the existing approach, the identified best routes for utilizing practical implementation from ambulance station to Parlama Mebrat accident site and to the nearest emergency hospital covers $5 \mathrm{~km}$ which takes 3.7 minutes.
\end{abstract}

in two minute's service area and $7 \mathrm{~km}$ which takes 6 minutes in four minute's service area. To sum up, the overall traversing physical distance is about $12 \mathrm{~km}$ and 9.7 minutes of travelling time. As per section 3.1.4, the estimated cost of traversing on the selected route to address emergency service is about 436.5 minutes and 1660.26 birr annually as shown below in table 13 .

Table 13. Existing annual traveling time cost analysis for PM accident site.

\begin{tabular}{|c|c|c|c|c|c|c|c|c|c|}
\hline \multirow{2}{*}{ AS } & \multirow{2}{*}{ HC } & \multicolumn{2}{|c|}{ Best Route from AS - AS - HC } & \multirow{2}{*}{$\mathbf{F}$} & \multirow{2}{*}{$\begin{array}{l}\text { DP (Oct 12, } \\
\text { 2012) }\end{array}$} & \multirow{2}{*}{$\mathrm{L}=\mathrm{D} / 5.5 \mathrm{~km}$} & \multirow{2}{*}{$\mathrm{STP}=\mathrm{L} * \mathrm{DP}$} & \multicolumn{2}{|c|}{ Cost in terms of } \\
\hline & & D (km) & $\mathbf{T}$ (min) & & & & & Time $=F^{*} \mathbf{T}$ & ETB $=\mathbf{F} *$ STP \\
\hline \multirow{2}{*}{ PM } & Zewuditu & 5 & 3.7 & 45 & 16.91 & 0.909 & 15.373 & 166.5 & 691.77 \\
\hline & Yekatit & 7 & 6 & 45 & 16.91 & 1.273 & 21.522 & 270 & 968.48 \\
\hline
\end{tabular}

III. Cost evaluation best route analysis for KM accident site

In the existing approach, the identified best routes for utilizing practical implementation from ambulance station to Kebena Mebrat accident site and to the nearest hospital (i.e., Menilik II Hospital) covers $6.8 \mathrm{~km}$ which takes 6.5 minutes to traverse. To sum up, the overall traversing physical distance is about $6.8 \mathrm{~km}$ and 6.5 minutes of travelling time. As per section 3.1.4, the estimated cost of traversing on the selected route to address emergency service is about 390 minutes and 1254.06 birr.

Table 14. Existing annual travelling time cost analysis for KM accident site.

\begin{tabular}{|c|c|c|c|c|c|c|c|c|c|}
\hline \multirow{2}{*}{ AS } & \multirow{2}{*}{$\mathrm{HC}$} & \multicolumn{2}{|c|}{ Best Route from AS - AS - HC } & \multirow{2}{*}{$\mathbf{F}$} & \multirow{2}{*}{$\begin{array}{l}\text { DP (Oct 12, } \\
\text { 2012) }\end{array}$} & \multirow{2}{*}{$\mathrm{L}=\mathrm{D} / \mathbf{5 . 5 \mathrm { km }}$} & \multirow{2}{*}{$S T P=L * D P$} & \multicolumn{2}{|c|}{ Cost in terms of } \\
\hline & & D (km) & $T$ (min) & & & & & Time $=F^{*} \mathbf{T}$ & ETB $=F *$ STP \\
\hline KM & Menlilik II & 6.8 & 6.5 & 60 & 16.91 & 1.236 & 20.901 & 390 & 1254.06 \\
\hline Total & & 6.8 & 6.5 & 60 & 16.91 & 1.236 & 20.901 & 390 & 1254.06 \\
\hline
\end{tabular}

\subsubsection{Cost Analysis Comparison}

This study has come up with two approaches for cost comparisons in specific and in general terms. For specific comparison considering Semen Hotel Mebrat as a selected accident site, the estimated travelling time to address the accident site is about 210/645.66 and 312/857.85 minutes/birr for proposed and existing methods respectively. Accordingly, the difference $b / n$ the two for Semen Hotel Mebrat accident site is 102/212.19 minutes/birr. This implies that, the proposed approach minimize travelling cost by reducing 102/212.19 minutes/birr from the existing one. Moreover, it minimizes the benefits of ambulance service by reducing 6.5/290.76 minutes/Birr and 90/221.01 minutes/birr for Parlama Mebrat and Kebena Mebrat respectively. For Cumulative comparison considering all accident sites as a selected accident location, the estimated travelling time to address Semen Hotel Mebrat, Parlama Mebrat and Kebena Mebrat accident sites is about 870/3048.20 and 1138.50/3772.16 minutes/birr for proposed and existing methods respectively. Accordingly, the difference $\mathrm{b} / \mathrm{n}$ the two for all accident sites is 268.5/723.96 minutes/birr. This implies that, the current study come up with a total reducing travelling duration and cost by 268.5/723.96 minutes/birr respectively from the existing one annually. For more detail information see the following common best route cost comparison table.

Table 15. Annual traveling time cost analysis comparison.

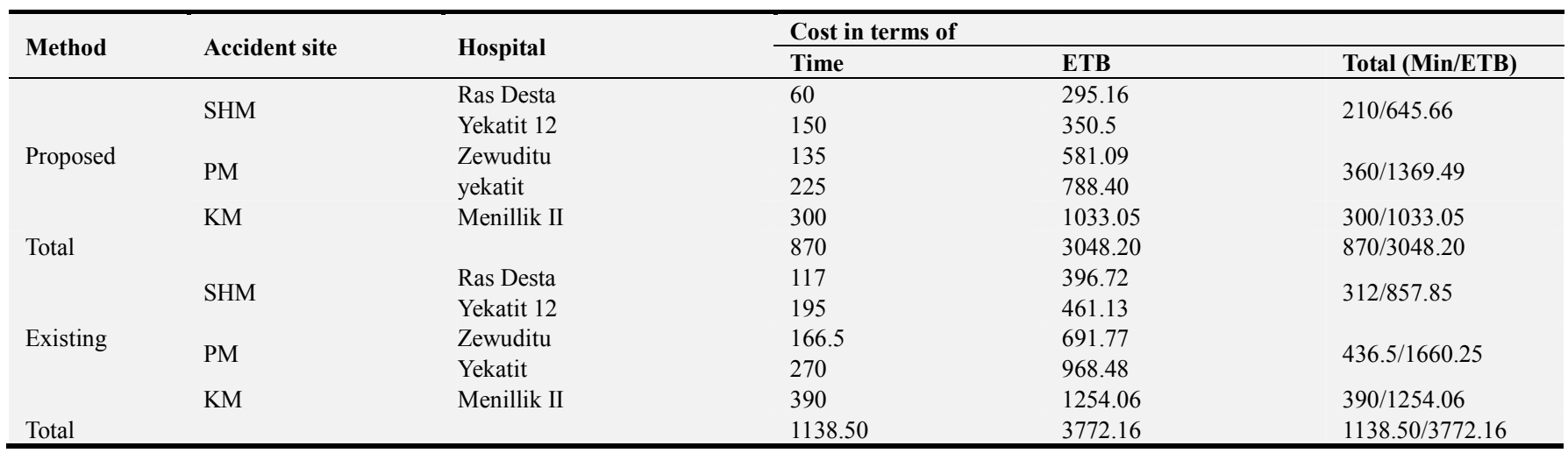

\subsection{Discussions}

The overall emergency ambulance transportation service in Arada sub-city is generally is not good in service delivery status especially during peak hours. This circumstance is affected by so many inter-related factors. To mention some, the road conditions, traffic volume and ambulance efficiencies are worth to mention. In addition to this, there is no GIS-based organized geo-database data handling method 
for efficient ambulance transportation service management system. This implies that, the whole routine activities (related to ambulance transportation service) are done based on manually. Beyond it is tedious and time consuming, the responsiveness rate of efficient ambulance transportation system to the injured person is low and insufficient; this leads to more losses from lives and damages. Therefore, the current option used in this study focuses on utilization of network analysis in GIS environment for the betterment of ambulance transportation service by designing GIS database layers along with their tabular attributes. For instance, roads (Name, Length, Speed limit, One-way, Category, Hierarchy); accident (A_ID, A_Name); hospital (H_ID, H_Name, address); ambulance station (Stn_ID, Stn_Name, address) were entered during the database designing stage in Arc Map GIS software. Accordingly, the analysis can perform easily by finding incidents (black spots), facilities (hospitals) and quickest routes (generated routes). For instance; in the case of road congestion or other incidences the efficient ambulance transportation service is interrupted. During this time, best route selection scheme can easily trace the best route which is economically viable within minute interval from the ambulance station to accident location; and from there to the nearest emergency hospital. Accordingly, the current study indicates that, it is possible to address highly emergent accidents in the sub-city if there are additional black spots and health care centers. Besides, the capacity of the existing ambulances to travel on every possible road links is another opportunity for the benefit of emergency ambulance service in the sub-city. For rational implementations, the study come up with two approaches (existing and proposed). In the existing approach $66 \%$ of health centers are found in four minutes service area domain however, $100 \%$ of health centers are found in the same service area domain in the proposed approach. Furthermore, the estimated travelling time and cost to address all accident sites of the selected sample best routes is about $870 / 3048.20$ and 1138.5/3772.16 minutes/birr for proposed and existing methods respectively.

\section{Conclusions}

The study revealed that, making different service area scenario (i.e., 1, $2 \& 4$ minutes service area domains which resembles to very urgent, moderate urgent and low urgent service areas) and selecting the associated best routes for each scenario could maximize response time and contribute to higher survival rates from severe remedial events in Arada sub-city. It is difficult to analyze problems faced by the ambulance transportation service on the road network without GIS. Therefore the study proves that, GIS has the ability to capture, store, analyze and display the information's which interrupts the ambulance service and send the routing information's to the ambulance driver. In other words, GIS plays an important role in decision making process of ambulance transportation service in Arada sub city to be easier, accurate and time effective.
Geo-database is highly vital for efficient ambulance transportation service management system. The attribute data of the road network, the turn table, the existing ambulance station, black spots and health centre data's are stored in an organized manner in ArcGIS environment. Besides, the developed network datasets are so important entities for further analysis in network analysis environment. The study also revealed that, road network analysis in GIS environment is sufficient enough to delineate the service area domains within the given impedance value. This demonstrates how accessibility varies with impedance as one goes from the concentric service areas. Moreover, the road network analysis is also essential to select the best routes from the ambulance station to the incident site; and from there to the nearest emergency health centers. This analysis minimizes the total cost of traveling time of efficient ambulance transportation service in the sub city. It also provides route information's about driving direction that helps how the ambulance drivers will drive through the routes. In general, the study revealed that, health center service area domain is increased by $34 \%$ from the existing technique while using service area analysis whereas duration and cost is decreased by $268.5 / 723.96$ minutes/birr while using best route analysis.

\section{References}

[1] B. Ganeshkumar, D. (2010). Emergency Response Managment and Information System a GIS based software to resolve the emergency recovery challenges in Madurai city, Tamil Nadu. International Journal of Geomatics and Geoscinces volume 1, No 1, 2010, 1-11.

[2] World Health Organization Fact Sheet, (2011). Road Traffic Injury.

[3] Moore. D, (2002). GIS in Emergency Planning, a spatial analysis of ambulance services.

[4] Addis Ababa city road authority section. (2004/2006). Addis Ababa urban transport study (AAUTS). Addis Ababa, Ethiopia.

[5] Addis Ababa city CSA. (2007). Federal Democratic Republic of Ethiopia Central Statistical Agency, statistical abstract. Addis Ababa, Ethiopia.

[6] Addis Ababa City Administration Master Plan Study Section. (2004). newly formed sub city and kebeles under the new structure of Addis Ababa City. Unpublished material. Addis Ababa, Ethiopia.

[7] Mauricio, p. S. (2003). Geographic information System and Intelligent Transportation Systems: Technologies used to form new communication networks.

[8] Mass et al. (1998). Incident Management and Intelligent Transportation Systems Technology: Estimating benefits for Northern verginia, the intelligent transportation society of America: USA.

[9] Arada sub city command post section. (2011/2012). Ambulance transportation service follows up standardized document and annual report. Unpublished material. Addis Ababa, Ethiopia. 
[10] Moses Santhakumar, S. s. (1998). Proceedings on Advanced computer Applications in transportation Engineering and planning, Regional Engineering College, Tiruchirappalli.

[11] Lo, C. P., Albert K. W. Yeung. (2002). Concepts and Techniques of Geographic Information Systems. New Jersey: Prentice Hall-Hall, Inc. 212-216.

[12] Godchild, M. (1998). Geographic Information Systems and Disaggregate Transportation Modeling Geographical Systems. Pp. 19-44.
[13] Imtiyaz, Pasha. (2006). Ambulance management System using GIS. Sweeden: Linkoping University.

[14] ASC crime \& traffic accident investigation office. (20072011). Arada Sub city traffic accident statistical report. Unpublished source. Addis Ababa, Ethiopia.

[15] ESRI. (1996). ArcView Network Analyst, Optimum Routing, Closest Facility and service Area Analysis. Environmental Systems Research institute, INC: USA. 